\title{
Moving away from PPARs - EGFR signaling and the anti- cancer effects of thiazolinedinediones
}

\author{
Jesse Roman ${ }^{1}$ \\ ${ }^{1}$ Division of Pulmonary, Allergy and Critical Care Medicine, Department of Medicine, Emory University School of Medicine, and \\ Veterans Affairs Medical Center, Atlanta, Georgia 30322, USA \\ Cell Research (2009) 19:669-671. doi: 10.1038/cr.2009.62; published online 1 June 2009
}

It is estimated that there were over 12 million new cases of cancer worldwide in 2007. Of these, lung cancer remains the number one cause of cancer death in the U.S. and many other countries. Current therapy for lung cancer involves multimodality therapies. Unfortunately, many patients are or become refractory to therapy and develop drug resistance. Kras and epidermal growth factor receptor (EGFR) mutations represent some of the most common mutations in lung cancer and have been implicated in chemoresistance. Therefore, despite advances in the detection and treatment of this disease, the overall 5 -year survival rate remains less than $15 \%$ [1]. Advances in the management of this deadly condition can come not only from strategies for early diagnosis, but also from new insights into disease pathogenesis that may lead to more effective means of treatment.

The thiazolidinediones represent an interesting group of agents with modest anti-cancer activity demonstrated both in vitro and in animal models [2]. Recently, a retrospective analysis of data obtained from the Veterans Integrated Services Network 16 data warehouse

\footnotetext{
Correspondence: Jesse Roman

Whitehead Biomedical Research Building, 615 Michael St, Suite 205-M, Atlanta, Georgia, 30322, USA

Tel: 404-712-2970; Fax: 404-712-2974

E-mail: jroman@emory.edu
}

revealed that the use of thiazolidinediones was associated with reduced risk of lung cancer [3]. These studies suggest that elucidating the mechanisms responsible for the anti-cancer effects of thiazolidinediones might inform our efforts for the development of new anticancer strategies.

The thiazolidinediones are a class of antidiabetic drugs that includes pioglitazone, rosiglitazone, ciglitazone, and troglitazone. Earlier work revealed that thiazolidinediones work by targeting peroxisome proliferator-activated receptors (PPAR) [4]. PPARs are ligandactivated transcription factors belonging to the nuclear hormone receptor superfamily that control energy homeostasis and cell differentiation, proliferation, apoptosis, and inflammation. Early research also linked PPARs to carcinogenesis and, to date, PPARs have been implicated in solid organ cancers like breast, ovary, prostate, bladder, gastric, colon, and lung cancers. Among the three subtypes (PPAR $\alpha$, PPAR $\beta / \delta$, and PPAR $\gamma$ ), the role of PPAR- $\gamma$ has been investigated the most in carcinogenesis mainly because of the availability of thiazolidinediones, which are known to stimulate PPAR $\gamma$ effectively; this is the reason these agents are also referred to as PPAR $\gamma$ ligands.

By acting on PPAR $\gamma$, thiazolidinediones inhibit tumor progression through effects on cellular differentiation, apoptosis induction, and growth ar- rest [4]. However, several investigators have found that thiazolidinediones have cellular effects that are independent of PPAR $\gamma$ activation. In fact, thiazolidinediones have been shown to inhibit tumor growth in mice injected with PPAR $\gamma$-deficient embryonic stem cells [5]. These so called PPAR $\gamma$-independent effects of thiazolidinediones include induction of apoptosis by inhibition of the survival functions of $\mathrm{Bcl}-2 / \mathrm{Bcl}-$ $\mathrm{XL}$, effects on mitochondrial respiratory functions, inhibition of translation initiation, and induction of tumor suppressors [2, 4]. Thus, elucidating the PPAR $\gamma$-independent anti-cancer effects of thiazolidinediones may prove beneficial.

In a report published in this issue of Cell Res, Li and colleagues showed that troglitazone inhibits the proliferation of endothelial cells and several types of carcinoma cells through another PPAR $\gamma$ independent pathway that entails the inhibition of EGFR signaling [6]. This study not only adds to an increasing number of manuscripts exploring the anti-cancer effects of thiazolidiones, but also contributes to our understanding of carcinogenesis in general, and dissects PPAR $\gamma$-independent events responsible for this activity.

The choice of troglitazone for this study is an interesting one considering that this drug was removed from the market in 2000 because of hepatotoxicity. Despite this, its use in the laboratory 
continues to unveil useful clues about cell cycle control and carcinogenesis. The authors observed in porcine aorta endothelial cells that troglitazone induced the phosphorylation of Extracellular Signal-Regulated Kinases (Erk), and used this system to explore the mechanisms responsible for the cellular effects of troglitazone. Importantly, they showed that troglitazone had the strongest Erk inductive effect, while other thiazolidinediones (i.e., ciglitazone, rosiglitazone and pioglitazone) had a lower effect with pioglitazone being the least efficient. Astutely, they noted that this ranking was different than that expected if PPAR $\gamma$ was involved in the process, since earlier studies established a different predicted ranking order for PPAR $\gamma$-thiazolidinedione binding (Rosi $>$ Pio $>$ TGZ > Cig) [7]. They also showed that a chemical inhibitor of PPAR $\gamma$ and the transfection of cells with a dominant negative PPAR $\gamma$ DNA construct failed to inhibit the effects of troglitazone. Finally, they found that troglitazone exerted similar effects in LNCaP cells, which are deficient in PPAR $\gamma$. Together, these complementary studies provide strong evidence that the phenomenon studied was independent of PPAR $\gamma$ activation. The studies also provide a good strategy for testing the role of PPAR $\gamma$ when defining intracellular signals responsible for the effects of PPAR $\gamma$ ligands.

The group went on to show that troglitazone binds to EGFR and induces its internalization through Grb2, followed by induction of Erks. Interestingly, Erk activation was not involved in troglitazone-induced cell growth inhibition; instead, regulation of Akt activation appeared important. The authors concluded that troglitazone attenuated overactive EGFR signaling by inhibition of Akt phosphorylation and induction of effective endo-lysosomal degradation of the receptor leading to growth arrest.

Although many of the findings reported by Li et al. were obtained using pulmonary aorta endothelial cells, the authors extended their work to carcinoma cells, which allowed for a very intriguing observation; in essence, that not all carcinoma cells tested showed inhibition of proliferation when exposed to troglitazone. Specifically, A549 (a lung epithelial cancer cell line) and Du145 (a prostate epithelial carcinoma cell line) cells showed decreased proliferation, whereas Hela (cervical epithelial carcinoma) and A431 (epidermoid carcinoma) cells did not. Consistent with their contention that EGFR mediated the effects of troglitazone, inhibition of cell growth was only demonstrated in cell lines that also showed increased EGFR degradation, but not in the ones that did not.

The observation that troglitazone targets EGFR is an important one, particularly in view of the role EGFR plays in lung and other cancers. Interestingly, EGFR signaling has also been implicated in the cellular effects of rosiglitazone. Furthermore, in A549 cells, rosiglitazone potentiated the anti-proliferative effects of gefitinib, an EGFR inhibitor available for clinical use in the treatment of several cancers [8].

Although a key message of this manuscript is that troglitazone can exert anti-cancer effects independent of PPAR $\gamma$ activation, it is important to note that PPAR $\gamma$ and EGFR signaling appear to intersect in some cell types. For example, in urothelium, PAPR $\gamma$ regulation of urothelial differentiation is modulated by downstream EGFR signaling. This has led others to propose that a balance between PPAR $\gamma$ and EGFR signaling might be required for a cell to switch from differentiation to proliferation and vice versa [9].

Together, the above observations suggest that troglitazone, through PPAR $\gamma$ independent signals that target EGFR, might serve as a reasonable complement to current cancer therapeutic regimens. Interestingly, EGFR inhibitors such as gefitinib show antitumor activity in only a subset (10-20\%) of non-small cell lung cancer patients having mutated EGFR. One wonders if these patients might benefit from therapies that include troglitazone. Yet, more information is needed before strong conclusions are drawn regarding its usefulness in the clinic. This is further highlighted by several seemingly contradictory reports showing that EGFR inhibitors may protect against thiazalinedinedioneinduced cell death in osteoblastic cells, that troglitazone induces EGFR transactivation in certain cells, and that troglitazone exerts its anti-cancer effects in breast cancer-derived cells not through EGFR signaling, but mainly through alterations in the $\mathrm{Na} / \mathrm{H}$ exchanger resulting in intracellular acidosis [10]. Clearly, thiazolidinediones have many effects other than PPAR $\gamma$ activation; the elucidation of such mechanisms holds the promise of unveiling new targets for the development of new anti-cancer therapies.

\section{References}

1 Spiro SG, Porter JC. Lung Cancer Where are we today? Current advances in staging and nonsurgical treatment. Am J Respir Crit Care Med 2002; 166:1166-1196.

2 Blanquicett C, Roman J, Hart CM. Thiazolidinediones as anti-cancer agents. Cancer Ther 2008; 6:25-34.

3 Govindarajan R, Ratnasinghe L, Simmons DL, et al. Thiazolidinediones and the risk of lung, prostate, and colon cancer in patients with diabetes. J Clin Oncology 2007; 25:1476-1481.

4 Roman J. Peroxisome proliferatoractivated receptor gamma and lung cancer biology: implications for therapy. J Investig Med 2008; 56:528-533.

5 Palakurthi SS, Aktas H, Grubissich LM, Mortensen RM, Halperin JA. Anticancer effects of thiazolidinediones are independent of peroxisome proliferatoractivated receptor gamma and mediated by inhibition of translation initiation. Cancer Res 2001; 61:6213-6218.

6 Li X, Yang X, Xu Y, et al. Troglitazone inhibits cells proliferation by attenuation of epidermal growth factor receptor signaling independent of peroxisome proliferator-activated receptor $\gamma$. Cell 
Res 2009; 19:720-732.

7 Willson TM, Brown PJ, Sternbach DD, Henke BR. The PPARs: from orphan receptors to drug discovery. J Med Chem 2000; 43:527-5550.

8 Lee SY, Hur GY, Jung KH, et al. PPAR $\gamma$ agonist increase gefitinib's antitumor activity through PTEN expression. Lung Cancer 2006; 51:297-301.

9 Varley CL, Stahlschmidt J, Lee WC, et al. Role of PPARgamma and EGFR signaling in the urothelial termina differentiation program. J Cell Sci 2004; 117:2029-2036.
10 Friday E, Oliver R 3rd, Welbourne T, Turturro F. Role ofEGFR signaling versus cellular acidosis via $\mathrm{Na} / \mathrm{H}$ exchanger (NHE1)-inhibition in troglitazoneinduced arrest of breast cancer-derived cells MCF-7. Cell Physiol Biochem 2007; 20:751-762. 